\title{
Beneficial Effects of a Synbiotic Supplement on Self-Perceived Gastrointestinal Well-Being and Immunoinflammatory Status of Healthy Adults
}

\author{
Esther Nova, ${ }^{1}$ Blanca Viadel, ${ }^{2}$ Julia Wärnberg, ${ }^{1}$ Jose E. Carreres, ${ }^{2}$ and Ascensión Marcos ${ }^{1}$ \\ ${ }^{1}$ Immunonutrition Group, Department of Metabolism and Nutrition, Institute of Science \\ and Technology of Food and Nutrition (ICTAN), Spanish National Research Council (CSIC), \\ Madrid; and ${ }^{2}$ Department of New Products, Ainia Center of Technology, Valencia, Spain
}

\begin{abstract}
The use of synbiotics as health promoters is still poorly defined, and human intervention studies are scarce. This study was designed to evaluate the effects of a commercialized synbiotic product containing Lactobacillus acidophilus La5, Bifidobacterium animalis ssp. lactis Bb-12, Lactobacillus delbrueckii ssp. bulgaricus, Lactobacillus paracasei ssp. paracasei, Streptococcus thermophilus, and fructooligosaccharides on the self-reported gastrointestinal well-being and the immunoinflammatory status of healthy human subjects. In this randomized, double-blind, placebo-controlled study, 20 women and 16 men (25-45 years old) received either three tablets per day of the synbiotic product $\left(2.4 \times 10^{9}\right.$ colony-forming units/day) or placebo during 6 weeks. Gastrointestinal symptoms and bowel habits were evaluated through a self-administered questionnaire. In those subjects suffering from any kind of digestive disturbance (mild dyspepsia, flatulence, postprandial bloating, constipation, etc.), improvements in symptoms after product consumption were also evaluated. Blood lymphocyte subsets, phagocytic activity, serum C-reactive protein, ceruloplasmin, and adhesion molecules concentrations were analyzed prior and after treatment. A significant improvement in overall self-reported gastrointestinal symptoms and bowel habit was found in the synbiotic group. A marginal effect of treatment (analysis of variance $P=.050$ ) was observed with L-selectin, which showed a significant decrease in the synbiotic group $(P=.019)$. In addition, basal L-selectin levels correlated with final intercellular adhesion molecule (ICAM)-1 levels $(r=0.468 ; P=.050)$, and basal ICAM-1 levels tended to correlate negatively with final L-selectin concentration $(r=-0.457 ; P=.056)$. None of these correlations was found in the placebo group. The rest of the immunological parameters studied were not modified by the intervention. In conclusion, consumption of the synbiotic product improves self-perceived bowel habits and might facilitate a better profile of adhesion molecules in healthy adults.
\end{abstract}

KEY WORDS: $\bullet$ adhesion molecules $\bullet$ bowel habits $\bullet$ gastrointestinal symptoms $\bullet$ immunomodulation $\bullet$ inflammatory proteins • lymphocyte subsets $\bullet$ probiotic bacteria

\section{INTRODUCTION}

$\mathbf{S}_{\mathrm{P}}$ YNBIOTIC PREPARATIONS CONTAINING a combination of probiotics and fermentable fiber (prebiotic) are considered a healthy dietary supplement in the restoration and maintenance of colonic flora. Probiotics are defined as live microorganisms that when administered in adequate amounts confer a health benefit on the host. ${ }^{1}$ Microorganisms that are principally used as probiotics include various species of lactobacilli or bifidobacteria, used individually or in combination, whereas the most used prebiotic fibers include inulin and fructooligosaccharides (FOS). Many human studies confirm that FOS have a bifidogenic effect on human colonic endogenous flora. ${ }^{2}$ Moreover, because the prebiotic

Manuscript received 31 December 2008. Revision accepted 5 July 2010.

Address correspondence to: Esther Nova, Department of Nutrition and Metabolism Institute of Science and Technology of Food and Nutrition, CSIC, C/Jose Antonio Novais 10, 28040 Madrid, Spain,E-mail: enova@if.csic.es fibers are preferential substrates for bifidobacteria, adding the prebiotic molecules to the probiotics would increase the survival rate of the bifidobacteria during intestinal transit and thus their effect on the colon. ${ }^{3,4}$ However, because probiotics do not permanently colonize the intestine, they must be taken in sufficient quantities to maintain adequate amounts in the colon. The principal purported healthpromoting effects of probiotics are the enhancement of mucosal immune defenses, with an adjuvant effect, increased polymeric immunoglobulin A production, and cytokine stimulus, ${ }^{5-7}$ resulting in modulation of systemic immunity, which adds to the general mechanisms conferring a protective effect against pathologic microbial colonization and translocation. ${ }^{8}$ The major effects claimed for the prebiotic components in functional foods include improved bowel function, increased mineral absorption, and a reduced risk of cardiovascular disease associated with dyslipidemia, insulin resistance, obesity, and possibly type 2 diabetes and of colon cancer. ${ }^{9}$ 
The use of synbiotics as health promoters is still poorly defined, and studies with combinations of pro- and prebiotics are still scarce. The effects of synbiotic therapies on intestinal function of different types of critically ill patients have been investigated in a few studies including infants ${ }^{10}$ and adults. ${ }^{11,12}$ Synbiotics have also proved useful in short bowel syndrome, ${ }^{13,14}$ ulcerative colitis,${ }^{15-17}$ and acute pancreatitis, ${ }^{18}$ although some of these are only preliminary studies without a control group.

A substantial amount of work has been published with the probiotic strains Lactobacillus acidophilus La5 and Bifidobacterium animalis ssp. lactis Bb12 present in the synbiotic product used in our study. The combination of the strains L. acidophilus La5, B. animalis ssp. lactis Bb12, and yogurt culture starters with or without prebiotics has been assessed in several conditions, such as Helicobacter pylori infection ${ }^{19}$ and critically ill patients admitted into the intensive care unit. ${ }^{11}$ The results suggest that the supplements provide a favorable alteration of the microbial composition of the upper gastrointestinal tract.

Based on the lack of studies assessing the usefulness of synbiotics in healthy individuals, the main objective of this study was to evaluate the effects of a synbiotic product containing five lactic acid bacterial strains and FOS on selfperceived gastrointestinal well-being and immunoinflammatory status of healthy adults. Second, in those subjects suffering from any kind of digestive disturbance such as mild dyspepsia, flatulence, postprandial bloating, constipation, etc., improvements in symptoms after product consumption were also evaluated.

\section{MATERIALS AND METHODS}

\section{Study population}

Healthy adults $(n=42) 25-45$ years old were recruited in the technological park of Valencia, Spain, for a pilot study with the synbiotic product. The sample size was chosen in order to provide enough statistical power for immune parameter changes on the basis of the data reviewed in the literature..$^{20-23}$ The exclusion criteria were the following: to be a vegetarian or to be on a diet, to suffer from any diagnosed gastrointestinal disease, chronic diseases such as diabetes and/or dyslipidemia, have had intestinal resections, or receiving chronic pharmacological treatment or acute antibiotic treatment 2 weeks prior to the intervention. Taking these criteria into account, 37 volunteers were finally included in this study who were judged to be in good health on the basis of physical examination and medical history. The volunteers provided written informed consent prior to the start of the study, and it was conducted in accordance with the ethical rules of the Helsinki Declaration $\left(52^{\text {nd }}\right.$ World Medical Association General Assembly, held in Edinburgh, United Kingdom, in October 2000), and the EEC Good Clinical Practice guidelines (document 111/3976/88 in July 1990). One volunteer did not complete the study, thus leaving 18 subjects in each study group with a female:male ratio of 10:8 in both groups. The study was ap- proved by the Ethics Committee of Virgen de los Lirios Hospital, Alcoy, Spain.

\section{Study design}

In this double-blind study, the subjects were randomly allocated to one of two groups matched by sex and age and received either three placebo capsules per day (containing sucrose, talcum powder, and stearic acid magnesium salt) or three tablets per day of the synbiotic product $\left(\right.$ SYMBYO ${ }^{\circledR}$, Laboratorios Phergal S.A., Torrejon de Ardoz, Spain). Each tablet contained a combination of five bacterial strains in approximately equal numbers (L. acidophilus La5, B. animalis ssp. lactis $\mathrm{Bb}-12$, Lactobacillus delbrueckii ssp. bulgaricus, Streptococcus thermophilus, and Lactobacillus paracasei ssp. paracasei [Probio-Tec ${ }^{\circledR}$, Chr. Hansen, Hørsholm, Denmark]) and FOS. The tablet was developed to be taken with thorough chewing, whereas the polysaccharide matrix allowed the formation of a protective gel in acid $\mathrm{pH}$ that improved survival of the bacteria until the intestine was reached. The consumption of three tablets per day accounted for a total daily consumption of $2.4 \times 10^{9}$ colony-forming units and $1.4 \mathrm{~g}$ of FOS. The subjects were advised to consume the tablets after their three main meals. They were also asked to continue with their normal diet, but they were not allowed to consume any other fermented dairy products, prebiotic, probiotic, or synbiotic supplement, or fiberenriched products during the study and 2 weeks prior to the intervention. They were only allowed to eat non-dairy fermented products, such as dry/cured meat, beer, or wine, if that was their previous habit. The intervention was conducted for 6 weeks. Compliance with the product or placebo consumption was good as assessed in the final questionnaire.

In the early morning 12-hour fasting venous blood samples were collected at the start of the study (T1) and 6 weeks later at the end of the examination period (T2). Before the specimens were obtained a questionnaire was administered in order to exclude alterations of immune parameters due to any current illness, such as allergy or infection. Blood samples were always analyzed for hematological and immunological variables within 4-5 hours after they were obtained. Basic anthropometric measurements (body weight, height, and waist circumference) were performed on the volunteers at the same visits.

Lifestyle, dietary habits, and self-reported gastrointestinal well-being were assessed at baseline through a questionnaire including questions on food intake frequency, physical activity, smoking habit, gastrointestinal symptoms (constipation, diarrhea, flatulence, postprandial bloating, lactose intolerance, and dyspepsia), and respiratory infections, all relative to the previous 2 months. Each of these symptoms was verbally explained to all subjects in order to standardize the evaluation criteria. These symptoms were explained as a simplification of the Rome II diagnostic criteria as follows: dyspepsia as pain or discomfort centered in the upper abdomen; postprandial bloating as a feeling of abdominal fullness or bloating after eating; constipation as 
two or more of the following-frequency of stools lower than three times a week, stools that are hard and lumpy, necessity to strain during defecation, and necessity to use laxatives; diarrhea as a high frequency of loose or watery stools (more than three-fourths of the time); and alternating diarrhea/constipation, as both conditions tend to appear in alternation and/or combined with periods of normal function. Moreover, lactose intolerance was explained as the feeling of stomach sickness after eating foods with lactose in them, accompanied or not by other symptoms such as bloating, gas, or diarrhea. Finally, flatulence was described as an excessive passage and expulsion of gas through the intestine that can be bothersome. In the end-point questionnaire the subjects were asked to evaluate subjectively, through one closed question (improvement: yes or no) any alleviation in previously reported symptoms and also any improvement in their bowel habits regarded as regularity of defecations over the 6-week intervention period. The number of stools per day at the end of the treatment was also measured as a recall estimate in a time frame covering the last 2 weeks. The volunteers filled out a 24-hour dietary recall questionnaire administered in three different occasions: at the start, midpoint, and end of the study, with the last one carried out on a holiday.

\section{Analysis of hematological and immunological parameters}

Hematology was analyzed in whole blood samples collected into EDTA using an automated analyzer (Sysmex XE2100, Roche Diagnostics, Basel, Switzerland), including red blood cell counts, hemoglobin, hematocrit, red blood cell corpuscular indices, white blood cell counts, differential, and platelets. Lymphocyte subsets were analyzed by flow cytometry $\left(\right.$ FACScan $^{\circledR}, \mathrm{BD}$, Sunnyvale, CA, USA). Whole blood was incubated with monoclonal antibodies for CD3 (mature T cells), CD4 (helper cells), CD8 (cytotoxic/ suppressor cells), and CD56+CD16 (natural killer cells) following the manufacturer's instructions (BD Biosciences, San Jose, CA, USA). The double staining procedure (CD3/CD4, CD3/CD8, and CD3/CD56+CD16) was used. Phagocyte function was quantitatively assessed using a Phagotest kit (BD Biosciences) and flow cytometry. Inflammatory proteins such as highly sensitive C-reactive protein and ceruloplasmin were measured in serum by immunoturbidimetry (model AU2700 biochemistry analyzer, Olympus, Rungis, France). The serum adhesion moleculessoluble intercellular adhesion molecule (ICAM)-1, soluble vascular cell adhesion molecule (VCAM)-1, and soluble Lselectin-were analyzed with commercial enzyme-linked immunosorbent assay kits (Diaclone, Besançon, France). The sensitivities of these kits were less than $0.1 \mathrm{ng} / \mathrm{mL}$ for soluble ICAM-1, less than $0.6 \mathrm{ng} / \mathrm{mL}$ for soluble VCAM-1, and less than $1 \mathrm{ng} / \mathrm{mL}$ for soluble L-selectin.

\section{Statistical analysis}

All data are expressed as mean and SD values. The statistical analysis of symptoms and its self-reported improve- ment was assessed by means of contingency tables and the Monte-Carlo exact test (two-tailed $P$ value was consigned). The effect of the synbiotic on hematological and immunological variables was assessed by one-way analysis of variance repeated-measures. When the analysis of variance test was significant, pairwise comparisons by Student's $t$ test (paired) were performed to further analyze the changes induced by the intervention within each group. A two-way analysis of variance repeated-measures was also performed in order to check any possible sex by group interaction. Between-group differences were analyzed both prior to and after the intervention by Student's $t$ test. Values of $P<.05$ were considered significant. All statistical analyses were performed using SPSS version 13.0 software (SPSS, Inc., Chicago, IL, USA).

\section{RESULTS}

No differences were observed in height (control group, $166.3 \pm 7.1 \mathrm{~cm}$; synbiotic group, $167.7 \pm 9.6 \mathrm{~cm} ; P=.620)$, weight (control group, $64.25 \pm 10.98 \mathrm{~kg}$; synbiotic group, $66.66 \pm 10.99 \mathrm{~kg} ; P=.514$ ), or body mass index (control group, $23.06 \pm 2.32 \mathrm{~kg} / \mathrm{m}^{2}$; synbiotic group, $23.74 \pm 2.19$ $\mathrm{kg} / \mathrm{m}^{2} ; P=.493$ ). In addition, no significant sex by group interaction in these anthropometric variables was found. Regarding evaluation parameters, no differences were observed between the groups in the basal values of any of them (frequency of each gastrointestinal symptom or respiratory infections, $P$ between .338 and 1.0; hematological variables, $P$ between .117 and .881 ; lymphocyte subsets, $P$ between .113 and .881 ; phagocytic capacity, $P=.908$; inflammatory proteins, $P=.227$ for C-reactive protein and $P=.958$ for ceruloplasmin; and adhesion molecules, $P$ between .204 and .487), which means that the placebo and the synbiotic groups are comparable in all relevant parameters prior to the intervention.

Table 1 presents the frequency of several gastrointestinal symptoms and infections in each group as obtained from the data reported by the subjects in the questionnaire previous to the treatment and also the changes reported in these symptoms following treatment. A total of 17 subjects reported suffering from gastrointestinal symptoms or frequent respiratory infections: eight in the placebo group and nine in the synbiotic group. The main difference between groups was found for the improvement in bowel habits, which was reported significantly more in the synbiotic group than in the placebo group (eight out of 18 in the synbiotic group and none in the placebo group). The number of stools per day showed a nonsignificant trend towards higher values in the synbiotic group $(P=.082)$; however, because the number of stools per day was not assessed prior to the intervention we cannot rule out that the trends already existed basally. Regarding the sum of symptoms, the cumulative self-reported improvement in any symptom was significantly more frequent in the synbiotic than in the placebo group $(P<.05)$. Diet seems not to be accountable for these effects because no differences were observed in the mean energy and 
Table 1. Basal Frequency and Postintervention Improvement in the Gastrointestinal Symptoms and Respiratory Infections in Healthy Subjects Receiving Placebo or Synbiotic Supplementation

\begin{tabular}{|c|c|c|c|c|c|c|}
\hline & \multicolumn{2}{|c|}{ Presence of symptom } & \multirow{2}{*}{$\begin{array}{c}\text { Monte-Carlo } \\
\text { exact test } \mathrm{P}\end{array}$} & \multicolumn{2}{|c|}{ Self-reported improvement } & \multirow{2}{*}{$\begin{array}{c}\text { Monte-Carlo } \\
\text { exact test } \mathrm{P}\end{array}$} \\
\hline & Placebo $(\mathrm{n}=18)$ & Synbiotic $(\mathrm{n}=18)$ & & Placebo & Synbiotic & \\
\hline Dyspepsia & $2 / 18$ & $2 / 18$ & 1.0 & $0 / 2$ & $0 / 2$ & - \\
\hline Postprandial bloating & $1 / 18$ & $3 / 18$ & .603 & $0 / 1$ & $1 / 3$ & 1.0 \\
\hline Lactose intolerance & $0 / 18$ & $1 / 18$ & 1.0 & - & $1 / 1$ & - \\
\hline Flatulence & $3 / 18$ & $4 / 18$ & 1.0 & $1 / 3$ & $4 / 4$ & .143 \\
\hline Chronic diarrhea & $0 / 18$ & $0 / 18$ & - & - & - & - \\
\hline Constipation & $1 / 18$ & $4 / 18$ & .338 & $0 / 1$ & $3 / 4$ & .400 \\
\hline Alternating diarrhea/constipation & $2 / 18$ & $0 / 18$ & .486 & $0 / 2$ & - & - \\
\hline Frequent respiratory infections & $2 / 18$ & $2 / 18$ & 1.0 & $0 / 2$ & $1 / 2$ & 1.0 \\
\hline Sum of symptoms & $\begin{array}{c}{[0,1,2]^{\mathrm{a}}} \\
(n=9,7,2)\end{array}$ & $\begin{array}{c}{[0,1,2]^{\mathrm{a}}} \\
(n=8,5,5)\end{array}$ & .565 & $\begin{array}{c}{[0,1]^{\mathrm{b}}} \\
(n=8,1)\end{array}$ & $\begin{array}{c}{[0,1]^{\mathrm{b}}} \\
(n=3,7)\end{array}$ & $<.05$ \\
\hline Bowel habits & - & - & & $0 / 18$ & $8 / 18$ & .003 \\
\hline Number of stools/day after treatment & & $\begin{array}{c}\text { Placebo } \\
{[0,1,2,3]^{\mathrm{c}}} \\
(n=3,15,0,0)\end{array}$ & & $\begin{array}{c}S y \\
{[0,} \\
(\mathrm{n}=1\end{array}$ & $\begin{array}{l}\text { tic } \\
3]^{\mathrm{c}} \\
4,1)\end{array}$ & $\begin{array}{l}\mathrm{P} \\
.082\end{array}$ \\
\hline
\end{tabular}

\footnotetext{
a The coding used is $0=$ no symptoms, $1=$ one symptom, and $2=$ two or more symptoms.

${ }^{\mathrm{b}}$ The coding used is $0=$ no symptom improved and $1=$ one or more symptoms improved.

${ }^{\mathrm{c}}$ The coding used is $0=$ no stools per day, $1=$ one stool per day, $2=$ two stools per day, amd $3=$ more than two stools per day.
}

macronutrient and fiber intakes between the synbiotic and the placebo groups (Table 2), and no differences were found either between the three different 24-hour recall questionnaires performed in weeks 0,3 , and 6 . During the study period six subjects took occasionally some kind of cold symptom relievers or pain relievers (such as ibuprofen or acetylsalicylic acid); however, three of them belonged to each study group.

Tables 3 and 4 show the results obtained for the immunological parameters measured. No significant interaction of time by group was observed in any hematological (data not shown) or immunological parameter except the serum concentration of L-selectin, which showed a significant decrease in the synbiotic group $(P=.019)$, whereas no differences were observed between the basal and final measurements in the placebo group $(P=.783)$. Regarding, however, the comparison of the final values observed in both groups, no differences were found in L-selectin. On the other hand, a significantly higher $\mathrm{CD}^{+}{ }^{+} \mathrm{CD} 4^{+}$percentage was observed in the synbiotic group than in the placebo group $(P=.003)$, but this was not reflected in the $\mathrm{CD}^{+} \mathrm{CD}^{+}$counts, which were similar between groups $(P=.180)$.

All adhesion molecules showed significant correlations between basal and final values for both all subjects together or split by treatment groups. Moreover, ICAM-1 and VCAM-1 showed correlation with each other. When correlations were analyzed in the two groups defined by treatment, it was observed that in the synbiotic group the basal L-selectin level correlated with final ICAM-1 level $(r=0.468 ; P=.050)$ and the basal ICAM-1 level correlated negatively with final L-selectin concentration $(r=-0.457$;

Table 2. Energy and Macronutrient Intakes in Healthy Subjects Receiving Placebo or Synbiotic Supplementation

\begin{tabular}{|c|c|c|c|}
\hline & Placebo & Synbiotic & $\mathrm{P}$ \\
\hline Energy (Kcal) & $2,122 \pm 420$ & $1,935 \pm 274$ & .127 \\
\hline Total protein $(\%)$ & $17.95 \pm 4.28$ & $18.48 \pm 4.39$ & .720 \\
\hline Vegetable protein $(\%)$ & $4.50 \pm 1.20$ & $4.89 \pm 0.86$ & .284 \\
\hline Animal protein $(\%)$ & $13.44 \pm 4.79$ & $13.59 \pm 4.80$ & .929 \\
\hline Total fat $(\%)$ & $43.56 \pm 5.49$ & $41.87 \pm 4.86$ & .340 \\
\hline SFA $(\%)$ & $14.65 \pm 3.10$ & $13.77 \pm 3.01$ & .400 \\
\hline MUFA (\%) & $17.48 \pm 3.83$ & $16.94 \pm 33.58$ & .665 \\
\hline PUFA $(\%)$ & $7.99 \pm 2.58$ & $8.05 \pm 2.40$ & .943 \\
\hline Total carbohydrates (\%) & $36.87 \pm 6.75$ & $38.26 \pm 7.20$ & .561 \\
\hline Soluble sugars $(\%)$ & $17.42 \pm 4.36$ & $17.68 \pm 6.01$ & .887 \\
\hline Starch $(\%)$ & $19.50 \pm 4.62$ & $20.58 \pm 6.80$ & .603 \\
\hline Fiber $(\mathrm{g})$ & $17.29 \pm 4.62$ & $16.33 \pm 5.93$ & .597 \\
\hline
\end{tabular}

Data are mean \pm SD values. Data were obtained by pooling the results of the three 24-hour recall questionnaires filled in during the study (basal, 3 weeks, and 6 weeks). All macronutrient intakes (except fiber) are expressed as a percentage of total energy intake.

MUFA, monounsaturated fatty acid; PUFA, polyunsaturated fatty acid; SFA, saturated fatty acid. 
Table 3. Lymphocyte Subsets in Healthy Subjects Receiving Placebo or Synbiotic Supplementation

\begin{tabular}{|c|c|c|c|c|c|}
\hline & \multicolumn{2}{|c|}{ Placebo } & \multicolumn{2}{|c|}{ Synbiotic } & \multirow[b]{2}{*}{$A N O V A \mathrm{P}$} \\
\hline & $T 1$ & $T 2$ & $T 1$ & $T 2$ & \\
\hline \multicolumn{6}{|c|}{ Helper T cells $\left(\mathrm{CD}^{+}{ }^{+} \mathrm{CD} 4^{+}\right)$} \\
\hline$\%$ & $39.42 \pm 7.29$ & $40.85 \pm 5.91$ & $44.00 \pm 9.18$ & $47.50 \pm 6.14^{\#}$ & .418 \\
\hline Cells $/ \mu \mathrm{L}$ & $830 \pm 356$ & $873 \pm 367$ & $1,015 \pm 437$ & $1,087 \pm 366$ & .744 \\
\hline \multicolumn{6}{|c|}{ Cytotoxic $\mathrm{T}$ cells $\left(\mathrm{CD} 3^{+} \mathrm{CD} 8^{+}\right)$} \\
\hline$\%$ & $27.11 \pm 7.49$ & $27.31 \pm 6.54$ & $25.19 \pm 6.02$ & $25.42 \pm 5.19$ & .953 \\
\hline Cells $/ \mu \mathrm{L}$ & $564 \pm 214$ & $585 \pm 258$ & $576 \pm 282$ & $584 \pm 212$ & .995 \\
\hline \multicolumn{6}{|c|}{$\mathrm{NK}$ cells CD3 ${ }^{-}(\mathrm{CD} 56+16)^{+}$} \\
\hline$\%$ & $14.27 \pm 7.62$ & $13.08 \pm 6.55$ & $11.69 \pm 5.08$ & $10.59 \pm 4.12$ & .804 \\
\hline Cells $/ \mu \mathrm{L}$ & $287 \pm 160$ & $278 \pm 187$ & $253 \pm 126$ & $233 \pm 93$ & .767 \\
\hline CD4/CD8 (cells $/ \mu \mathrm{L})$ & $1.59 \pm 0.58$ & $1.58 \pm 0.45$ & $1.85 \pm 0.60$ & $1.96 \pm 0.52$ & .500 \\
\hline
\end{tabular}

Data are mean \pm SD values. No significant differences were found between treatment groups in $\mathrm{T} 1$.

${ }^{\#}$ Significant differences between treatment groups after the intervention (T2) by Student's $t$ test: $P<.01$.

ANOVA, analysis of variance; NK, natural killer; T1, prior to treatment; T2, after 6 weeks of synbiotic consumption.

$P=.056)$. In contrast, no significant correlations were observed between L-selectin and ICAM-1 in the placebo group. The correlations found in the synbiotic group suggest that the decrease in L-selectin seems to occur in those patients who previously showed the highest levels of ICAM-1. In fact, if the subjects in the synbiotic group are considered in two groups according to those showing higher or lower values than $455 \mathrm{ng} / \mathrm{mL}$ in $\mathrm{T} 1$ ( $n=9$ in each group), the relative change in the group with higher values is a decrease of the $17 \%$ from basal values, whereas in the group with lower ICAM-1 values the relative change in L-selectin is a decrease of only $3 \%$. The difference between these relative changes, however, does not reach statistical significance $(P=.092)$.

\section{DISCUSSION}

Debate is currently open in the scientific community concerning the benefits of probiotics and prebiotics for adults with normal, healthy intestinal ecosystems; however, because the microbiota has such an important role in the intestinal metabolism and natural defense of the host, a great value has been conferred on probiotic bacteria able to counteract environmental factors (such as antibiotic therapy), capable of improving the barrier function of the mucosa and the resistance to the invasion and proliferation of pathogenic species and of promoting the gut-associated lymphoid tissue. This study showed that a 6-week intervention in healthy adults with a synbiotic product containing $L$. acidophilus La5, B. lactis $\mathrm{Bb} 12$, L. delbrueckii ssp. bulgaricus, S. thermophilus, L. paracasei ssp. paracasei, and FOS improves the self-reported bowel habits and gastrointestinal symptoms. The importance of these findings is limited by the intrinsic value of self-assessment, which involves some degree of subjective evaluation, with a possible recall bias. Despite this, self-assessment with diary recording of bowel movements is a tool that has been reported in the literature. ${ }^{24}$ Another limitation of this evaluation is that the severity of the symptoms prior to the intervention was not assessed.

Regarding blood parameters, the serum concentration of L-selectin was modified by administration of the synbiotic. Both B. lactis $\mathrm{Bb} 12$ and L. acidophilus La5 have shown adhesion capacity to intestinal mucus from healthy children (31\% and $4 \%$, respectively), and it is believed that the adherence of probiotic strains to the mucosa is related to their

Table 4. Phagocytic Capacity and Inflammatory Proteins in Healthy Subjects Receiving Placebo or Synbiotic Supplementation

\begin{tabular}{|c|c|c|c|c|c|}
\hline & \multicolumn{2}{|c|}{ Placebo } & \multicolumn{2}{|c|}{ Synbiotic } & \multirow[b]{2}{*}{$A N O V A \mathrm{P}$} \\
\hline & $T 1$ & $T 2$ & $T 1$ & $T 2$ & \\
\hline Phagocytic capacity (\%) & $93.30 \pm 0.89$ & $91.33 \pm 2.38$ & $93.37 \pm 2.10$ & $90.84 \pm 3.28$ & .602 \\
\hline $\mathrm{CRP}(\mathrm{mg} / \mathrm{L})$ & $0.88 \pm 0.72$ & $0.90 \pm 0.68$ & $1.38 \pm 1.44$ & $1.24 \pm 1.28$ & .705 \\
\hline Ceruloplasmin (mg/dL) & $33.8 \pm 9.4$ & $30.5 \pm 4.0$ & $33.4 \pm 10.1$ & $33.9 \pm 10.7$ & .219 \\
\hline sICAM-1 (ng/mL) & $469 \pm 85$ & $433 \pm 82$ & $434 \pm 78$ & $406 \pm 50$ & .674 \\
\hline sVCAM-1 (ng/mL) & $615 \pm 212$ & $575 \pm 198$ & $545 \pm 126$ & $521 \pm 148$ & .732 \\
\hline sL-selectin (ng/mL) & $2,586 \pm 555$ & $2,560 \pm 626$ & $2,724 \pm 600$ & $2,389 \pm 420^{*}$ & .050 \\
\hline
\end{tabular}

Data are mean \pm SD values. No significant differences were found between treatment groups in $\mathrm{T} 1$ and $\mathrm{T} 2$.

*Significant differences between T1 and T2 values within groups by paired $t$ test: $P<.05$.

CRP, C-reactive protein; sICAM-1, soluble intercellular adhesion molecule-1; sL-selectin, soluble L-selectin; sVCAM-1, vascular cell adhesion molecule-1. 
immunomodulatory properties, ${ }^{25}$ which might help explain the effect on serum L-selectin levels in the current study. It is also important to underline that the synbiotic consumption in the conditions of this study did not bring any negative side effects to the volunteers.

The self-reported enhancement in bowel habits of the participating subjects is in agreement with previous reports on the effects of synbiotic administration on intestinal function of patients with gastrointestinal disease ${ }^{10,14}$ as well as in healthy subjects. ${ }^{26,27}$ This effect has been attributed to the role of short-chain fatty acids, such as acetate, propionate, and butyrate (end products of bacteria metabolism), in the large bowel. Although these fatty acids have been studied for their role in intestinal motility, ${ }^{28,29}$ considerably more studies have focused on butyrate for its role in nourishing the colonic mucosa and in the prevention of colon cancer ${ }^{30}$ and also for its influence on cytokine production by immune cells, which has produced so far controversial results. 31,32

L-selectin levels are measured in serum or plasma as a marker of the L-selectin levels in lymphocytes and neutrophils. One early phase of atherosclerosis involves the recruitment of inflammatory cells from the circulation and their transendothelial migration. Selectins (P, E, and L) and their ligands are involved in the rolling and tethering of leukocytes on the vascular wall, whereas ICAMs and VCAMs, as well as some of the integrins, induce firm adhesion of inflammatory cells at the vascular surface. ${ }^{33} \mathrm{Ex}-$ pression of VCAM-1, ICAM-1, and L-selectin has been consistently observed in atherosclerotic plaques. Thus, the decrease in L-selectin levels in the subjects consuming the synbiotic might lessen the magnitude of ongoing inflammatory processes. However, it should be considered that the effect of treatment was only marginally significant, and no differences were found in the final values of L-selectin between the synbiotic and the placebo groups, which reduces the importance of the pre-to-post within-group finding. On the other hand, it is worth noting that the subjects' L-selectin values were always within the normal range, and clearer benefits might occur in subjects with cardiovascular risk factors. The correlations observed seem to point out a regulatory mechanism affecting L-selectin and ICAM-1 levels that is favored by the synbiotic. This is relevant because there is accumulating evidence from prospective studies suggesting that elevated circulating levels of soluble ICAM1 might predict cardiovascular events in initially healthy people. ${ }^{34-36}$ In this regard, probiotic bacteria belonging to the species $L$. caseii have been shown to reduce leukocyte trafficking to an inflamed segment of bowel, because of decreased ICAM-1 expression on vascular cells in an experimental model of colitis. ${ }^{37}$ The effect of probiotics on adhesion molecules suggests an interaction of bacterial components present in the synbiotic with the gastrointestinal resident cells, such as epithelial and immune cells (macrophages, dendritic cells, and lymphocytes). Commensal bacteria have been shown in vivo to interact with intestinal epithelial cells and to be internalized through specialized cells, allowing the interaction with underlying immune tissues and the regulation of local immune responses involving modulation of cytokine secretion and receptor expression on the cell surface. ${ }^{38}$

This is a small study regarding the number of subjects included, which might have been a limitation in observing a more significant effect on L-selectin. This is in fact a pilot study that mainly serves to pinpoint possible health effects of this synbiotic, which are worth looking at in larger, hypothesis-driven studies, both in healthy subjects and in patients with cardiovascular disease.

In conclusion, this study shows that daily intake of the synbiotic supplement has beneficial effects on the selfperceived bowel habits of healthy subjects and might decrease the serum levels of L-selectin, possibly leading to a more beneficial profile of adhesion molecules regarding the prevention of atherosclerosis and cardiovascular disease.

\section{ACKNOWLEDGMENTS}

The authors thank the statistician Mrs. Laura Barrios, B.Sc. for her assistance with the statistical analysis. This work was supported by Laboratorios Phergal S.A.

\section{AUTHOR DISCLOSURE STATEMENT}

No competing financial interests exist.

\section{REFERENCES}

1. Food and Agriculture Organization/World Health Organization: Expert Consultation Report: Health and Nutritional Properties of Probiotics in Food Including Powder Milk and Live Lactic Acid Bacteria. Argentina. October 2001. www.fao.org/es/ESN/Probio/ report.pdf (accessed August 27, 2007).

2. Gibson GR, Roberfroid MB: Dietary modulation of the human colonic microbiota: introducing the concept of prebiotics. $\mathrm{J} \mathrm{Nutr}$ 1995;125:1401-1412.

3. Scherezenmeir J, De Vrese M: Probiotics, prebiotics and synbiotics-approaching a definition. Am J Clin Nutr 2001;73 (2 Suppl):361S-364S.

4. Duggan C, Gannon J, Walker WA: Protective nutrients and functional foods for the gastrointestinal tract. Am J Clin Nutr 2002;75:789-808.

5. Isolauri E, Majamaa H, Arvola T, Rantala I, Virtanen E, Arvilommi H: Lactobacillus casei strain GG reverses increased intestinal permeability induced by cow milk in suckling rats. Gastroenterology 1993;105:1643-1650.

6. Walker WA: Role of nutrients and bacterial colonization in the development of intestinal host defense. J Pediatr Gastroenterol Nutr 2000;30(Suppl 2):S2-S7.

7. Nova E, Toro O, Varela P, Lopez-Vidriero I, Morande G, Marcos A: Effects of a nutritional intervention with yogurt on lymphocyte subsets and cytokine production capacity in anorexia nervosa patients. Eur J Nutr 2006;45:225-233.

8. Lu L, Walker WA: Pathologic and physiologic interactions of bacteria with the gastrointestinal epithelium. Am J Clin Nutr 2001;73:1124S-1130S.

9. Roberfroid MB: Prebiotics and probiotics: are they functional foods? Am J Clin Nutr 2000;71(6 Suppl):1682S-1687S.

10. Kanamori Y, Hashizume K, Sugiyama M, Mortomi M, Yuki N, Tanaka R: A novel synbiotic therapy dramatically improved the 
intestinal function of a pediatric patient with laryngotracheoesophageal cleft (LTEC) in the intensive care unit. Clin Nutr 2002;21:527-530.

11. Jain PK, McNaught CE, Anderson AD, MacFie J, Mitchell CJ: Influence of synbiotic containing Lactobacillus acidophilus La5, Bifidobacterium lactis Bb 12, Streptococcus thermophilus, Lactobacillus bulgaricus and oligofructose on gut barrier function and sepsis in critically ill patients: a randomised controlled trial. Clin Nutr 2004;23:467-475.

12. Bengmark S: Use of some pre-, pro- and synbiotics in critically ill patients. Best Pract Res Clin Gastroenterol 2003;17:833-848.

13. Kanamori Y, Hashizume K, Sugiyama M, Morotomi M, Yuki N: Combination therapy with Bifidobacterium breve, Lactobacillus casei, and galactooligosaccharides dramatically improved the intestinal function in a girl with short bowel syndrome: a novel synbiotics therapy for intestinal failure. Dig Dis Sci 2001;46: 2010-2016.

14. Kanamori Y, Sugiyama M, Hashizume K, Yuki N, Morotomi M, Tanaka R: Experience of long-term synbiotic therapy in seven short bowel patients with refractory enterocolitis. J Pediatr Surg 2004;39:1686-1692.

15. Haskey N, Dahl WJ: Synbiotic therapy: a promising new adjunctive therapy for ulcerative colitis. Nutr Rev 2006;64:132-138.

16. Macfarlane S, Furrie E, Kennedy A, Cummings JH, Macfarlane GT: Mucosal bacteria in ulcerative colitis. Br J Nutr 2005; 93(Suppl 1):S67-S72.

17. Furrie E, Macfarlane S, Kennedy A, Cummings JH, Walsh SV, O’Neil DA, Macfarlane GT: Synbiotic therapy (Bifidobacterium longum/Synergy 1) initiates resolution of inflammation in patients with active ulcerative colitis: a randomised controlled pilot trial. Gut 2005;54:242-249.

18. Olah A, Belagyi T, Poto L, Romics L Jr, Bengmark S: Synbiotic control of inflammation and infection in severe acute pancreatitis: a prospective, randomized, double blind study. $\underline{\text { Hepato- }}$ gastroenterology 2007;54:590-594.

19. Wang KY, Li SN, Liu CS, Perng DS, Su YC, Wu DC, Jan CM, Lai CH, Wang TN, Wang WM: Effects of ingesting Lactobacillus- and Bifidobacterium-containing yogurt in subjects with colonized Helicobacter pylori. Am J Clin Nutr 2004;80:737-741.

20. Chiang BL, Sheih YH, Wang LH, Liao CK, Gill HS: Enhancing immunity by dietary consumption of a probiotic lactic acid bacterium (Bifidobacterium lactis HN019): optimization and definition of cellular immune responses. Eur J Clin Nutr 2000; 54:849-855.

21. Parra MD, Martínez de Morentin BE, Cobo JM, Mateos A, Martínez JA: Daily ingestion of fermented milk containing Lactobacillus casei DN114001 improves innate-defense capacity in healthy middle-aged people. J Physiol Biochem 2004;60:85-91.

22. Bartosch S, Woodmansey EJ, Paterson JC, McMurdo ME, Macfarlane GT: Microbiological effects of consuming a synbiotic containing Bifidobacterium bifidum, Bifidobacterium lac$t i s$, and oligofructose in elderly persons, determined by real-time polymerase chain reaction and counting of viable bacteria. Clin Infect Dis 2005;40:28-37.

23. Meyer AL, Micksche M, Herbacek I, Elmadfa I: Daily intake of probiotic as well as conventional yogurt has a stimulating effect on cellular immunity in young healthy women. Ann Nutr Metab 2006;50:282-289.

24. Mearin F, Badía X, Balboa A, Benavent J, Caballero AM, Domínguez-Muñoz E, Garrigues V, Piqué JM, Roset M, Cucala M, Figueras M; the RITMO Group: Predictive factors of irritable bowel syndrome improvement: 1-year prospective evaluation in 400 patients. Aliment Pharmacol Ther 2006;23:815-826.

25. Juntunen M, Kirjavainen PV, Ouwehand AC, Salminen SJ, Isolauri E: Adherence of probiotic bacteria to human intestinal mucus in healthy infants and during rotavirus infection. Clin Diagn Lab Immunol 2001;8:293-296.

26. Pregliasco F, Anselmi G, Fonte L, Giussani F, Schieppati S, Soletti L: A new chance of preventing winter diseases by the administration of synbiotic formulations. $\mathrm{J}$ Clin Gastroenterol 2008;42(Suppl 3 Pt 2):S224-S233.

27. De Paula JA, Carmuega E, Weill R: Effect of the ingestion of a symbiotic yogurt on the bowel habits of women with functional constipation. Acta Gastroenterol Latinoam 2008;38:16-25.

28. Cherbut C: Motor effects of short-chain fatty acids and lactate in the gastrointestinal tract. Proc Nutr Soc 2003;62:95-99.

29. Tazoe H, Otomo Y, Kaji I, Tanaka R, Karaki SI, Kuwahara A: Roles of short-chain fatty acids receptors, GPR41 and GPR43 on colonic functions. J Physiol Pharmacol 2008;59(Suppl 2):251-262.

30. Wong JM, de Souza R, Kendall CW, Emam A, Jenkins DJ: Colonic health: fermentation and short chain fatty acids. $\underline{J \text { Clin }}$ Gastroenterol 2006;40:235-243.

31. Nancey S, Bienvenu J, Coffin B, Andre F, Descos L, Flourié B: Butyrate strongly inhibits in vitro stimulated release of cytokines in blood. Dig Dis Sci 2002;47:921-928.

32. van Nuenen MH, de Ligt RA, Doornbos RP, van der Woude JC, Kuipers EJ, Venema K: The influence of microbial metabolites on human intestinal epithelial cells and macrophages in vitro. FEMS Immunol Med Microbiol 2005;45:183-189.

33. Blankenberg S, Barbaux S, Tiret L: Adhesion molecules and atherosclerosis. Atherosclerosis 2003;170:191-203.

34. Pradhan AD, Rifai N, Ridker PM: Soluble intercellular adhesion molecule-1, soluble vascular adhesion molecule-1, and the development of symptomatic peripheral arterial disease in men. Circulation 2002;106:820-825.

35. Malik I, Danesh J, Whineup P, Bhatia V, Papacosta O, Walker M, Lennon L, Thomson A, Haskard D: Soluble adhesion molecules and prediction of coronary heart disease: a prospective study and meta-analysis. Lancet 2001;358:971-976.

36. Luc G, Arveiler D, Evans A, Amouyel P, Ferrieres J, Bard JM, Elkhalil L, Fruchart JC, Ducimetiere P; PRIME Study Group: Circulating soluble adhesion molecules ICAM-1 and VCAM-1 and incident coronary heart disease: the PRIME Study. Atherosclerosis 2003;170:169-176.

37. Angulo S, Llopis M, Antolin M, Gironella M, Sans M, Malagelada JR, Piqué JM, Guarner F, Panés J: Lactobacillus casei prevents the upregulation of ICAM-1 expression and leukocyte recruitment in experimental colitis. Am J Physiol Gastrointest Liver Physiol 2006;291:G1155-G1162.

38. Corthésy B, Gaskins HR, Mercenier A: Cross-talk between probiotic bacteria and the host immune system. $J$ Nutr 2007;137 (3 Suppl 2):781S-790S. 